\title{
A Model of Self-Similar Data Traffic Applied to Ethernet Traces
}

\author{
J.J. Gordon* \\ CAPE Consulting Inc., Rumson, NJ 07760, U.S.A. \\ Tel+1-732-747-2479, Fax+1-732-741-1950,jjg@ capecons.com \\ F. Huebner-Szabo de Bucs* \\ AT\&T Labs, Holmdel, NJ 07733, U.S.A. \\ Tel+1-732-949-7639, Fax+1-732-949-1720, fhuebner@att.com
}

\begin{abstract}
This paper proposes a simple four-parameter model of self-similar data traffic. The basic element of the model is a single Pareto arrival stream, in which packet interarrival times (IATs) are iid Pareto distributed. The model proposes that two such streams be multiplexed or superimposed, to produce a composite stream in which the marginal IAT distribution is Pareto-like, and which exhibits power law correlation not only in block packet count (BPC) (the definition of self-similarity) but also in the IAT process. The presence of long range IAT correlations creates the potential for the more extreme queueing behavior associated with self-similar traffic.

We provide a simple parameter estimation procedure for the proposed model which involves the mean and variance of the IATs, the Hurst parameter $\mathrm{H}$, and the marginal IAT distribution. Based on analysis of Ethernet traces collected by Bellcore, the model is in good qualitative agreement with observed IAT distributions, and with the corresponding IAT and BPC autocorrelation functions. In simulated queueing studies, it reproduces the 'knee' in the mean delay curve for one Ethernet trace.

The proposed model exhibits the qualitative features present in self-similar Ethernet traffic: Pareto-like IAT marginals, and power law IAT and BPC correlations. It is conceptually simple, and easy to work with numerically (i.e., to simulate). It also provides physical insight into the nature of long range correlations, and the relationship between IAT and BPC correlations.
\end{abstract}

\section{Keywords}

Traffic Modeling, Self-Similarity, Pareto Distribution, Ethernet Traces

\footnotetext{
* Part of the work for this paper was done while the author was with Bellcore, Red Bank, N.J., U.S.A.

The original version of this chapter was revised: The copyright line was incorrect. This has been corrected. The Erratum to this chapter is available at DOI: 10.1007/978-0-387-35353-1_28 


\section{INTRODUCTION}

Aside from the packet length distribution, there are two factors that determine the queueing behavior of a packet stream entering a G/G/1 queue. The first is the marginal interarrival time (IAT) distribution, which is characterized by its mean and higher moments. The second is the correlation structure of the arrival process, which can be described directly by the IAT autocorrelation function $\rho_{\text {IAT }}(k)$, or indirectly by the autocorrelation function $\rho_{\mathrm{BPC}}(k)$ of the 'block packet count' (BPC) process. By definition, block packet counts $X_{k}$ are the numbers of packets arriving during consecutive fixed length intervals or 'blocks' of time. The time series $X_{k}$ is assumed here to be covariance stationary with autocorrelation function $\rho_{\mathrm{BPC}}(k)$. The functions $\rho_{\mathrm{IAT}}(k)$ and $\rho_{\mathrm{BPC}}(k)$ are clearly related, though not in a readily expressible form.

In classical queueing models based on e.g., phase-type distributions, the marginal IAT distribution is the main determinant of queueing behavior. In general, correlations are implicitly present in classical models. However, they decay exponentially fast and have only a second order impact on queueing. In contrast, long range or power law correlations are a fundamental property of self-similar traffic models. This type of correlation can in some cases have a more significant impact on queueing behavior than the variance and higher order moments of the IAT distribution. Given that selfsimilarity is present in data traffic from a variety of sources, self-similar traffic models are likely to play an important role in the design and cost-effective operation of future data networks.

Fractional Brownian Motion (FBM) [5] is perhaps the simplest self-similar traffic model. FBM abandons the traditional framework of point arrival processes in favor of modeling the aggregate work process (i.e., the BPC process). It incorporates selfsimilarity through the Hurst parameter $H$. However, in FBM $H$ is simply a parameter which is measured from data and plugged into the model. A more satisfactory model would derive the value of $H$ from more physically intuitive properties of data traffic. In addition to this philosophical criticism, BPC-based models of data traffic encounter a significant practical problem. The measurement of $H$ from BPC data appears to be a numerically unstable problem. To date, there are no reliable methods for extracting $\boldsymbol{H}$ from BPC data. Different BPC-based methods can produce markedly different $\boldsymbol{H}$ estimates [4]. Furthermore, even the same method can produce significantly different $H$ estimates, depending on the range of data considered, etc.

This paper proposes a self-similar traffic model based on multiplexed Pareto point arrival processes. This model has several advantages relative to FBM and other proposed models [6]-[8]. First, the proposed model appears to be in good agreement with real Ethernet data, based on analysis of the marginal IAT distribution and correlation structure. Second, the Pareto model provides insight into the nature of selfsimilarity. In the proposed model, long range BPC correlations are fundamentally due to power law tail of the marginal IAT distribution. When two Pareto traffic streams 
are multiplexed, some of the correlation is 'squeezed' out of the marginal's tail and into the IAT sequence itself. That is, the tail of the marginal IAT distribution becomes shorter, at the expense of introducing long range correlation into the multiplexed IAT sequence. This relationship between the IAT and BPC correlation structure may be a basic property of self-similar traffic.

Finally, based on this understanding of the relationship between IAT and BPC correlation, the proposed model provides a method of measuring the Hurst parameter $H$ from the more fundamental IAT autocorrelation function. This method of estimating $H$ appears to be far more reliable than methods based on BPC analysis.

\section{MULTIPLEXED PARETO MODEL}

As noted above, there are two properties of an arrival process that determine its queueing behavior through a G/G/1 queue: its marginal IAT distribution and correlation structure. Classical queueing models are typically concerned only with the marginal IAT distribution, which is characterized by its first, second and perhaps higher order moments. In self-similar models, due to the impact of long range correlations, it is also necessary to model the correlation structure of the arrival process. In the simplest formulation, this correlation structure is modeled solely through the Hurst parameter $H$, which occurs as an exponent in the power law decay of BPC correlations. For self-similar traffic, the asymptotic behavior of $\rho_{\mathrm{BPC}}(k)$ is $\rho_{\mathrm{BPC}}(k) \sim k^{2 H-2}$, where $k$ is the correlation lag and $1 / 2<H<1$.

A contention of this paper is that in order to satisfactorily model the correlation structure of an arrival process, it is necessary to use more than the single parameter $H$. Note that $H$ determines the asymptotic rate of decay of BPC correlations. It says nothing about the absolute magnitude of these correlations. If $\rho_{\mathrm{BPC}}(k)$ were plotted on log-log axes, the asymptotic slope of the plot would be $2 H-2$. However, it seems intuitive reasonable that the intercept of this plct should also have an impact on queueing. Furthermore, the BPC correlation structure is only half of the picture. In general, an arrival process will also exhibit IAT correlations, and it is a priori unclear whether IAT and BPC correlations should be modeled independently, or in some combined fashion.

The model proposed here models both IAT and BPC correlations in terms of two more fundamental parameters. This approach reflects the fact that IAT and BPC correlations are closely related, and that specifying one implicitly determines the other. The two parameters that model the correlation structure of the arrival process can be thought of as determining the slope and intercept of a log-log plot of $\rho_{\mathrm{BPC}}(k)$. Alternatively, they can be thought of as determining the slope and intercept of a log$\log$ plot of $\rho_{\mathrm{IAT}}(k)$. Both of these views are valid, and either one provides a basis for estimating the model parameters from data. The proposed self-similar model is based on the Pareto distribution, whose probability density function (PDF) and complementary cumulative distribution function (CDF) are defined as follows. 


$$
\begin{aligned}
& f(t)=\operatorname{Pr}[T=t]=\alpha \beta^{\alpha} /(\beta+t)^{\alpha+1} \\
& F_{c}(t)=\operatorname{Pr}[T>t]=\beta^{\alpha} /(\beta+t)^{\alpha}
\end{aligned}
$$

Note that the $k$ th moment of the density (1) is finite only for $k<\alpha$. In this paper we consider only cases for which $\alpha>1$, so that a finite mean exists. Consider a single Pareto stream in which IATs are iid distributed with density (1). In reference [1] it was shown that the Hurst parameter $H$ associated with this process is

$$
H=\left(\begin{array}{ll}
(1+\alpha) / 2 & 0<\alpha<1 \\
(3-\alpha) / 2 & 1<\alpha<2 \\
1 / 2 & \alpha>2
\end{array}\right.
$$

(Note that integer values of $\alpha$ require special treatment.) A single Pareto stream therefore provides a simple two-parameter model of self-similar traffic. A value of $H$ in the range $1 / 2<H<1$ maps into a unique value of $\alpha$ in the range $1<\alpha<2$, and the parameter $\beta$ would be chosen to match the mean arrival rate $\lambda$ : $\beta=(\alpha-1) / \lambda$.

As one might expect, this simple model is not sufficiently flexible to model real data. However, it does have two points in its favor. First, it suggests a 'physical' basis for self-similarity: long range correlations are a consequence of the power law tails of packet IAT distributions. Second, real packet data traces do indeed appear to have power law IAT distributions. Pareto-type processes are therefore attractive candidates for modeling self-similar data. The above points can be incorporated into a more sophisticated self-similar model. Consider a multiplexed stream of two Pareto processes, having parameters $\alpha_{1}, \beta_{1}$ and $\alpha_{2}, \beta_{2}$. In the component Pareto streams, IATs are iid distributed with density (1). In the combined stream, IATs will in general be correlated (i.e., no longer iid), and the marginal IAT distribution can be shown to have the following Pareto-like form

$$
\begin{aligned}
\hat{F}_{c}(t)= & \frac{\lambda_{1}}{\lambda_{1}+\lambda_{2}} \frac{\beta_{1}^{\alpha_{1}}}{\left(\beta_{1}+t\right)^{\alpha_{1}}} \frac{\beta_{2}^{\alpha_{2}-1}}{\left(\beta_{2}+t\right)^{\alpha_{2}-1}} \\
& +\frac{\lambda_{2}}{\lambda_{1}+\lambda_{2}} \frac{\beta_{1}^{\alpha_{1}-1}}{\left(\beta_{1}+t\right)^{\alpha_{1}-1}} \frac{\beta_{2}^{\alpha_{2}}}{\left(\beta_{2}+t\right)^{\alpha_{2}}}
\end{aligned}
$$

where $\lambda_{1}=\left(\alpha_{1}-1\right) / \beta_{1}$ and $\lambda_{2}=\left(\alpha_{2}-1\right) / \beta_{2}$. The first term in eqn. (4) is obtained by considering arrivals in stream 1 , and requiring that the IAT in stream 1 and the residual IAT in stream 2 both exceed $t$. The second term is obtained by applying the same requirements to stream 2 . In either stream, the residual IAT density 
$f_{R}(t)$ is equal to $F_{c}(t) \cdot \beta /(\alpha-1)$, where $F_{c}(t)$ is given by eqn. (2). (This is a standard result, discussed in [9].) Note that for large $t, \hat{F}_{c}(t) \sim t^{-\hat{\alpha}}$ where $\hat{\alpha}=\alpha_{1}+\alpha_{2}-1$. That is, the distribution (4) again has a power law tail, with the exponent being jointly determined by $\alpha_{1}$ and $\alpha_{2}$.

Multiplexing two Pareto traffic streams leads to a shorter tail of the composite marginal distribution at the expense of introducing long range correlation into the multiplexed IAT sequence. This effect is shown in Figure 1 in which the complementary distribution functions $F_{c}(t)$ of two Pareto traffic streams with $1<\alpha_{i}<2$ (dotted lines) and the corresponding $\hat{F}_{c}(t)$ of the multiplexed stream (solid line) are depicted. It can be clearly seen that the tail of the superimposed traffic stream is considerably shorter than the tails of the two individual streams. We suspect that this relationship between the shortening of heavy-tailed distributions (by multiplexing them) and the introduction of long range IAT correlations may be a basic property of self-similar traffic.

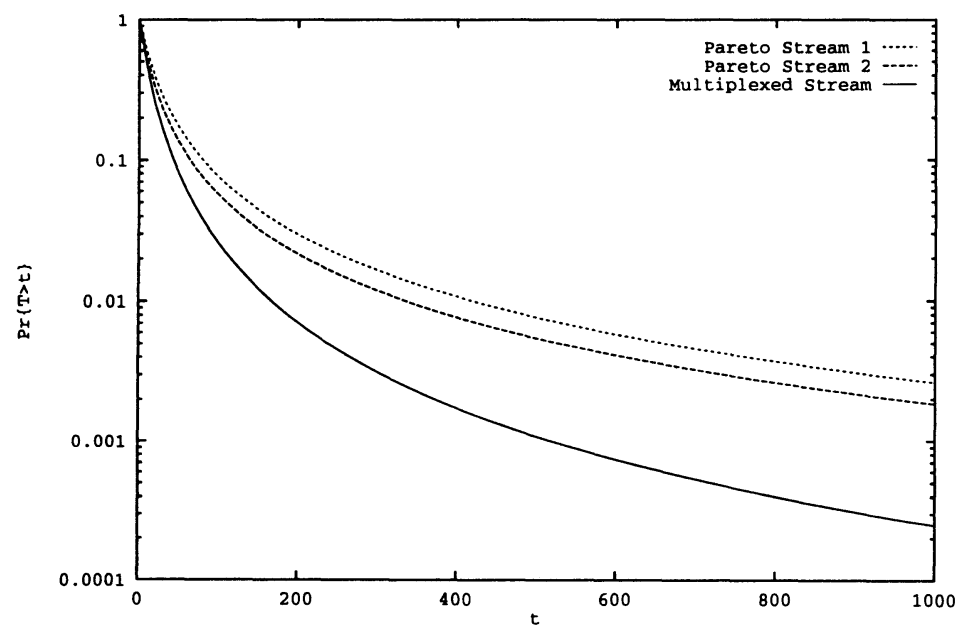

Figure 1 Tail behaviour of individual and multiplexed Pareto streams

In general, one cannot explicitly determine the autocorrelation functions $\hat{\rho}_{\mathrm{IAT}}(k)$ and $\hat{\rho}_{\mathrm{BPC}}(k)$ associated with the multiplexed stream. However, the following facts can be shown to hold. First, the Hurst parameter $\hat{H}$ in the multiplexed stream is equal to the larger of the Hurst parameters $H_{1}$ and $H_{2}$ associated with the component streams: Let $X_{k}^{(1)}$ and $X_{k}^{(2)}$ be the block packet counts in the component streams, and let $\rho_{\mathrm{BPC}}^{(1)}(k)$ and $\rho_{\mathrm{BPC}}^{(2)}(k)$ be the corresponding BPC autocorrelation functions. The BPC autocorrelation function in the multiplexed stream is 


$$
\begin{aligned}
\hat{\rho}_{\mathrm{BPC}}(k) & =\frac{\operatorname{cov}\left[X_{n+k}^{(1)}+X_{n+k}^{(2)}, X_{n}^{(1)}+X_{n}^{(2)}\right]}{\operatorname{var}\left[X_{n}^{(1)}+X_{n}^{(2)}\right]} \\
& =\frac{\operatorname{cov}\left[X_{n+k}^{(1)}, X_{n}^{(1)}\right]+\operatorname{cov}\left[X_{n+k}^{(2)}, X_{n}^{(2)}\right]}{\operatorname{var}\left[X_{n}^{(1)}\right]+\operatorname{var}\left[X_{n}^{(2)}\right]} \\
& =\omega_{1} \cdot \rho_{\mathrm{BPC}}^{(1)}(k)+\omega_{2} \cdot \rho_{\mathrm{BPC}}^{(2)}(k)
\end{aligned}
$$

where $\quad \omega_{1}=\operatorname{var}\left[X_{n}^{(1)}\right] / \operatorname{var}\left[X_{n}^{(1)}+X_{n}^{(2)}\right] \quad$ and $\omega_{2}=\operatorname{var}\left[X_{n}^{(2)}\right] / \operatorname{var}\left[X_{n}^{(1)}+X_{n}^{(2)}\right]$. It follows from $(5)$ that $\hat{\rho}_{\mathrm{BPC}}(k)$ will decay as the slower of $\rho_{\mathrm{BPC}}^{(1)}(k)$ and $\rho_{\mathrm{BPC}}^{(2)}(k)$, so that $\hat{H}=\max \left(H_{1}, H_{2}\right)$.

Finally, the power law decay of $\hat{\rho}_{\mathrm{IAT}}(k)$ can be related to $\hat{H}$ and $\hat{\rho}_{\mathrm{BPC}}(k)$ as follows. In [1] it was shown that in self-similar traffic, the mean arrival rate $\lambda(t)$ measured across a finite interval of time $[0, t]$ converges to its long term mean value $\lambda$ as $t^{2 H-2}$ :

$$
\lambda(t)-\lambda \sim t^{2 H-2}
$$

Assume that $\hat{\rho}_{\mathrm{IAT}}(k)$ is of the asymptotic form $k^{-\gamma}$. This assumption appears to be valid for real traffic (see section 3 ), and also for the model outlined above (see reference [2]). Based on results in [1], it follows that the mean IAT $\tau(n)$ across a finite sample of $n$ IATs converges to its long range mean value $\tau=\lambda^{-1}$ as $n^{-\gamma / 2}$ :

$\tau(n)-\tau \sim n^{-\gamma / 2}$

For consistency, the exponents in the power law convergence relations for $\lambda(t)$ and $\tau(n)$ in equations (6) and (7) must be identical. We therefore conclude that $\hat{H}$ is related to $\gamma$ via the following simple equation

$\hat{H}=1-\gamma / 4$

This equation expresses a basic relationship between the power law convergence behavior of $\hat{\rho}_{\mathrm{IAT}}(k)$ and $\hat{\rho}_{\mathrm{BPC}}(k)$. As explained in section 3, it forms a basis for estimating the Hurst parameter $H$ via analysis of IAT data. This method of $H$ estimation appears to be far more reliable than alternative methods based on direct analysis of BPC data. Note that equation (8) assumes $\hat{\rho}_{\mathrm{IAT}}(k)$ to be non-zero. This appears to be the case for real traffic. For single iid Pareto streams, in which $\hat{\rho}_{\mathrm{IAT}}(k) \equiv 0$ for $k>0$, equation (8) will still hold, but in a modified form. In that case, the infinite variance of the IAT distribution will ensure that $\tau(n)$ converges to its long term mean value according to a power law. 


\section{PARAMETER ESTIMATION PROCEDURE}

The multiplexed Pareto model described in section 2 contains four parameters: $\alpha_{1}$, $\alpha_{2}, \beta_{1}$, and $\beta_{2}$. The proposed method for estimating these parameters is as follows:

1. Plot the IAT autocorrelation function $\hat{\mathrm{P}}_{\mathrm{IAT}}(k)$ on $\log -\log$ axes and measure its asymptotic slope $\gamma$. The Hurst parameter $\hat{H}$ can then be calculated via equation (8).

2. Without loss of generality, assume that $\alpha_{1}<\alpha_{2}$ and $1<\alpha_{1}<2$. (Note that $\alpha_{2}$ could be greater than two.) Based on equation (3), and the comments at the end of section 2 , it follows that $\hat{H}=H_{1}>H_{2}$. The value of $\alpha_{1}$ is therefore given by:

$$
\alpha_{1}=3-2 \hat{H}=1+\gamma / 2
$$

3. Plot the IAT marginal distribution on $\log -\log$ axes, and measure its asymptotic slope $\eta$. This slope determines $\alpha_{2}$ via the relation:

$$
\alpha_{2}=\eta-\alpha_{1}+1
$$

4. Determine the ratio of the arrival rates $\lambda_{1}=\left(\alpha_{1}-1\right) / \beta_{1}$ and $\lambda_{2}=\left(\alpha_{2}-1\right) / \beta_{2}$ in the component Pareto streams via the equation $\lambda_{1} / \lambda_{2}=\kappa$. The parameters $\beta_{1}$ and $\beta_{2}$ can then be calculated in terms of the overall arrival rate $\lambda=\lambda_{1}+\lambda_{2}$ and the artificial parameter $\kappa$ :

$$
\begin{aligned}
& \beta_{1}=\left(\frac{1+\kappa}{\kappa}\right) \cdot \frac{\alpha_{1}-1}{\lambda} \\
& \beta_{2}=(1+\kappa) \cdot \frac{\alpha_{2}-1}{\lambda}
\end{aligned}
$$

5. Find the value of $\kappa$ which best fits the data. The recommended means of doing this is to select the value of $\kappa$ which matches the variance of the IAT distribution. In practice, this involves numerical iteration.

The above estimation procedure requires three degrees of freedom to match the Hurst parameter $\hat{H}$, and the mean and variance of the marginal IAT distribution. Note that $\hat{H}$ determines the slope of a log-log plot of $\hat{\rho}_{\mathrm{BPC}}(k)$ or $\hat{\rho}_{\mathrm{IAT}}(k)$. Ideally, one would like to use the fourth degree of freedom to match the intercept of these plots. Then two parameters would be used to fit the arrival process' IAT distribution, and two would be used to fit its correlation structure. However, directly matching the $\log -\log$ 
intercept of either $\hat{\rho}_{\mathrm{BPC}}(k)$ or $\hat{\rho}_{\mathrm{IAT}}(k)$ appears to involve numerical difficulties, and is not recommended here.

There are several criteria that could be used to determine $\kappa$ in step 5. For example, instead of matching the variance of the IAT distribution, one could instead select $K$ to match the asymptotic intercept of a log-log plot of the IAT distribution. Whatever method is employed, the fitted model should ideally reproduce both the asymptotic slope and intercept of $\hat{\rho}_{\mathrm{BPC}}(k)$ and $\hat{\rho}_{\mathrm{IAT}}(k)$. That is, it should accurately model both the asymptotic magnitude and rate of decay of $\hat{\rho}_{\mathrm{BPC}}(k)$ and $\hat{\rho}_{\mathrm{IAT}}(k)$. The Pareto model may be judged successful if it accurately reproduces these quantities.

\section{PARAMETER ESTIMATION - MARGINAL IAT DISTRIBUTION}

The above model was applied to three Ethernet traces which were collected by Bellcore, and which are described in detail in [3]. These traces can be assumed to be representative of LANs supporting text processing, email and scientific programming applications for many users. Traces 1-3 consisted of 968631,1359656 , and 643454 packet arrival events respectively, each representing one hour of recording time. Our analysis of these traces addresses the arrival process only. Packet length statistics are not considered.

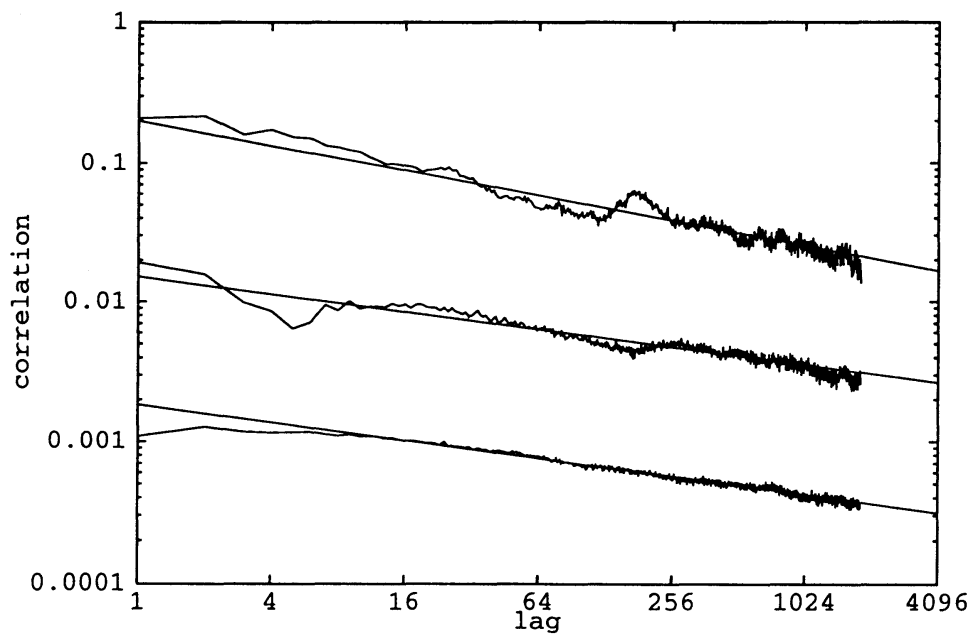

Figure 2 Interarrival time autocorrelation functions 
As described in section 3, the first step in fitting the model to each trace is to plot the IAT autocorrelation function $\hat{\rho}_{\mathrm{IAT}}(k)$. Figure 2 shows plots of $\hat{\rho}_{\mathrm{IAT}}(k)$ for traces $1-3$ on $\log -\log$ axes. Note that for clarity of presentation, traces 2 and 3 are offset from trace 1 on the $y$-axis by factors of 0.1 and 0.01 respectively. Also shown in Figure 2 are the linear approximations to $\hat{\rho}_{\mathrm{IAT}}(k)$. In each case, a least squares fit was obtained for lags $k=16$ to $k=1024$. The corresponding $\hat{H}$ estimates obtained from equation ( 8 ) were $0.92,0.95$, and 0.95 respectively.

This method of $\hat{H}$ estimation appeared to be far more straightforward and reliable than methods based on BPC analysis. Consider, for example, the 'log-variance' method of $\hat{H}$ estimation. This method requires one to plot the variance of the aggregated block packet counts $X_{k}^{(m)}=\left(X_{k m-m+1}+\ldots+X_{k m}\right) / m$ versus $m$ on $\log$-log axes. In self-similar traffic, the variance of $X_{k}^{(m)}$ decays asymptotically as $m^{2 \hat{H}-2}$. Consequently, one can in principle estimate $\hat{H}$ from the asymptotic slope of the log-variance plot.

In practice, this method appears to significantly under-estimate $\hat{H}$. Figure 3 shows $\log$-variance plots for traces 1-3. The $H$ estimates derived from these plots were 0.84 , 0.86 and 0.87 . When substituted into the Pareto model and simulated, these estimates produce significantly better queueing behavior than those derived from $\hat{\rho}_{\mathrm{IAT}}(k)$. Since the $\hat{\rho}_{\mathrm{IAT}}(k)$ estimates themselves appear to under-estimate the queue lengths obtained from the traces, we conclude that the log-variance method produces $\hat{H}$ estimates that are too conservative.

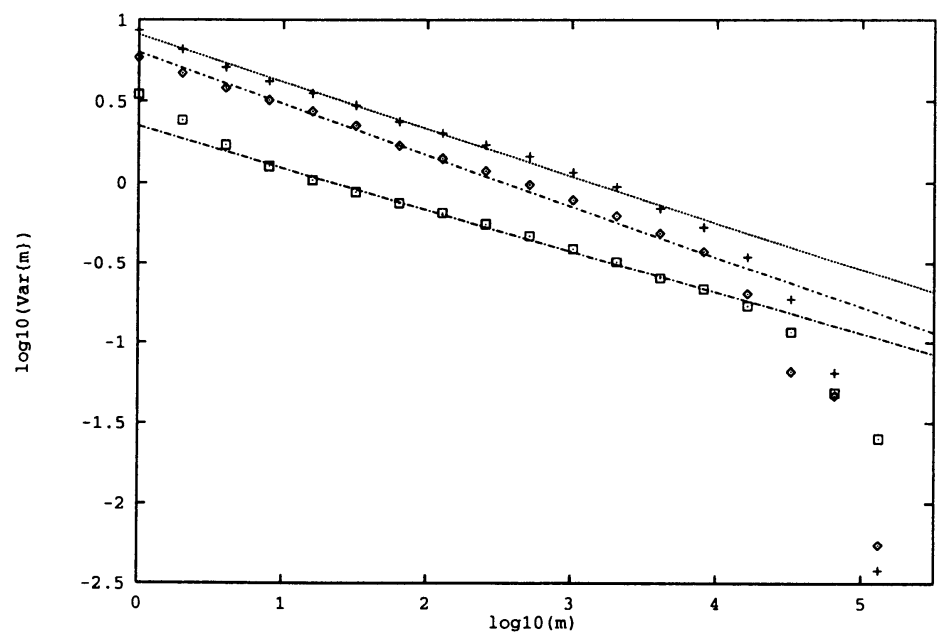

Figure 3 Log-variance plots 
Other BPC-based $\hat{H}$ estimates are more obviously unreliable. Figure 4 shows a plot of the periodogram of $\hat{\rho}_{\mathrm{BPC}}(k)$ for trace 1. For self-similar traffic, the periodogram should exhibit $1 / f$ noise. That is, it should diverge as $\omega^{2-2 \hat{H}}$ as the frequency $\omega \rightarrow 0$. In principle, this provides another method of estimating $\hat{H}$. However, the curve in Figure 4 is so variable or 'noisy' that no reliable estimate of $\hat{H}$ can be obtained. It appears that the BPC process is so volatile that it will not produce reliable $\hat{H}$ estimates over any realistic measurement interval. This problem affects all BPCbased methods of $\hat{H}$ estimation. For reasons that are not entirely clear, the problem does not affect IAT-based $\hat{H}$ estimation. Equation (8) therefore provides a more reliable basis for estimating $\hat{H}$.

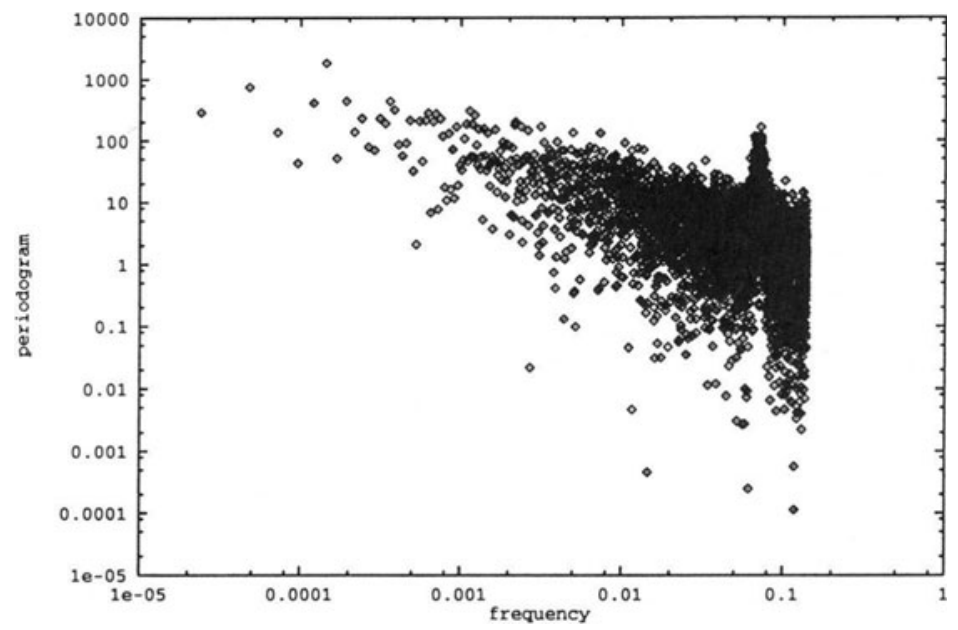

Figure 4 Block packet count periodogram

According to equation (9), the $\hat{H}$ estimates derived from Figure 2 imply the following values for $\alpha_{1}: 1.16,1.10$ and 1.10 . The next step in the estimation procedure is to calculate $\alpha_{2}$ from equation (10). Figure 5 shows plots of the marginal IAT distribution for each trace, plotted on log-log axes (solid lines). 


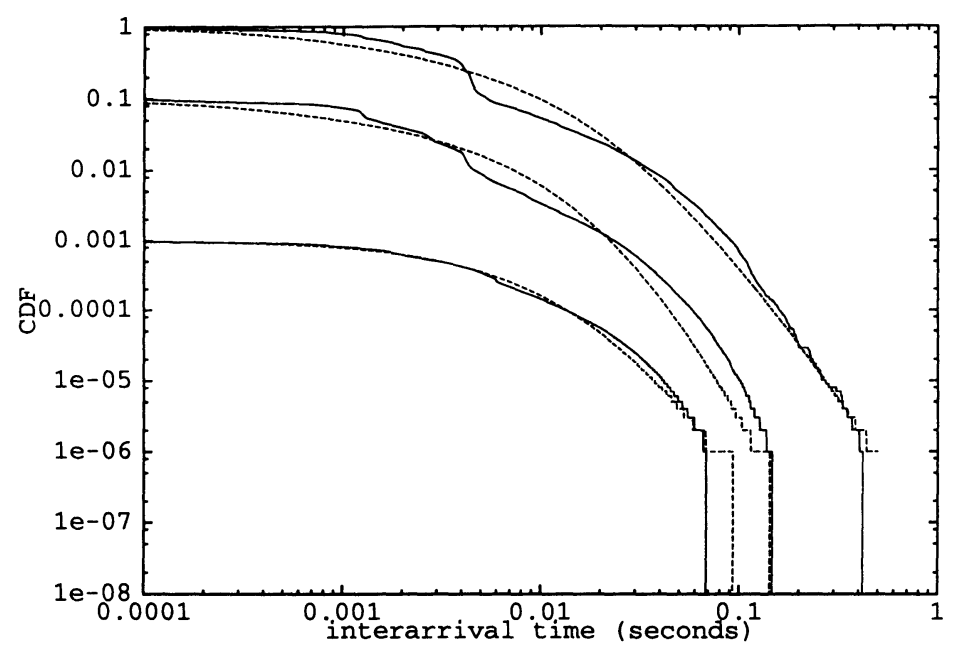

Figure 5 Marginal interarrival time distributions

Note that the function plotted in Figure 5 is the complementary distribution function $F_{c}(t)=\operatorname{Pr}[T>t]$. This function is more convenient to work with than the probability density function $f(t)=\operatorname{Pr}[T=t]$, since $F_{c}(t)$ integrates out the measurement 'noise' in $f(t)$. Also note that for clarity of presentation, the marginals for traces 2 and 3 are offset from trace 1 by factors of 0.1 and 0.001 respectively. The slope $\eta$ of the marginals' asymptotes is used to calculate $\alpha_{2}$ via equation (10). The $\alpha_{2}$ estimates obtained in this way are $\alpha_{2}=4.069,5.961$, and 4.397.

Given that estimates for $\alpha_{1}$ and $\alpha_{2}$ have been obtained, the final step in the parameter estimation procedure is to express $\beta_{1}$ and $\beta_{2}$ in terms of $\kappa$ and $\lambda$, as in equations (11) and (12), and find the value of $\kappa$ which matches the variance of the marginal IAT distribution. This involves numerical iteration on $\kappa$. The parameter values derived from this procedure are given in Table 1, together with the corresponding values of the mean IAT $\tau$, the IAT variance $\sigma^{2}$, and $\hat{H}$. The corresponding fitted marginal distributions, as given by equation (4), are superimposed on the actual distributions in Figure 5 (dotted lines). In all cases the Pareto model provides a good fit to the marginal IAT distribution. 
Table 1 Parameters for the Fitted Pareto Model

\begin{tabular}{cccc}
\hline Parameter & Trace 1 & Trace 2 & Trace 3 \\
$\hat{H}$ & 0.92 & 0.95 & 0.95 \\
$\tau(\mathrm{ms})$ & 3.716 & 2.646 & 5.594 \\
$\sigma^{2}\left(\mathrm{~ms}^{2}\right)$ & 51.323 & 21.680 & 63.202 \\
$\alpha_{1}$ & 1.16 & 1.10 & 1.10 \\
$\alpha_{2}$ & 4.069 & 5.961 & 4.397 \\
$\beta_{1}(\mathrm{~ms})$ & 1.046 & 0.514 & 3.108 \\
$\beta_{2}(\mathrm{~ms})$ & 26.420 & 27.061 & 23.174 \\
\hline
\end{tabular}

Several observations can be made regarding the fitted distributions. First, based on Figure 5 it appears that the fitted Pareto distributions are 'flatter' than the actual distributions i.e., less concave. A possible explanation for this is as follows. When a large number of Pareto processes are multiplexed, the resultant IAT distribution behaves like an exponential distribution for small $t$, while retaining a power law tail for large $t$. (The arrival process does not become Poisson, since long range correlations remain.) This type of distribution will have a more concave shape, as in Figure 5. It is possible that the Ethernet traffic is in practice generated by a large number of Pareto-like sources. Modeling this data in terms of just two Pareto sources is merely a useful mathematical approximation.

Second, the fitted distributions extend to infinity, whereas the actual distributions are truncated at some finite value of $t$. Note that in all cases, the point at which the actual distribution is truncated (i.e., becomes zero) is well out into the tail of the distribution. Trace 1, for example, has a mean IAT of 0.0042 seconds, while its IAT distribution extends out to 0.4 seconds. It follows that the fitted Pareto distributions model the actual distributions over a very large range of $t$, certainly several orders of magnitude beyond the mean.

Third, the plots in Figure 5 exhibit a jump or discontinuity at around $t \approx 0.0042$ seconds. The origin of the jump is unclear, though it may be related to Ethernet packet segmentation or acknowledgment times. Regardless of its origin, this jump has negligible impact on queueing. 


\section{PARAMETER ESTIMATION - CORRELATION STRUCTURE}

Figure 6 shows the IAT autocorrelation functions $\hat{\rho}_{\mathrm{IAT}}(k)$ obtained from the Pareto model (solid line with symbols), superimposed on the actual autocorrelation functions derived from the Ethernet traces. Also shown in Figure 6 are the linear approximations to the trace autocorrelation functions (solid lines without symbols). Note that for clarity of presentation, the plots for traces 2 and 3 are offset from trace 1 by factors of 0.1 and 0.01 respectively.

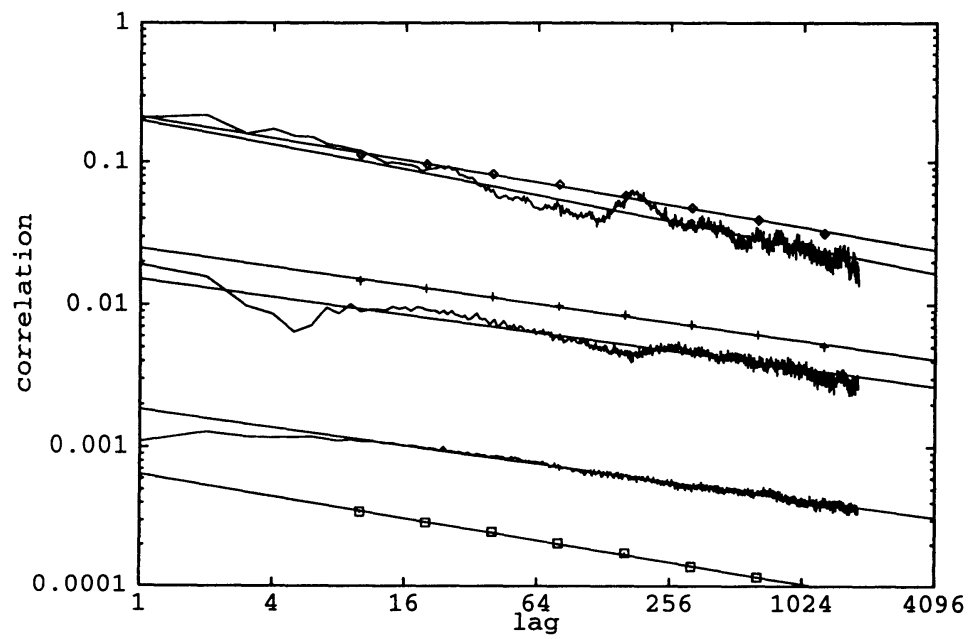

Figure 6 Interarrival time autocorrelation functions

Since $\hat{\rho}_{\mathrm{IAT}}(k)$ is difficult to calculate exactly for the Pareto model, the Pareto results in Figure 6 were obtained by simulation of the fitted Pareto model. For traces 1 and 2 the model is in reasonable agreement with the data, while for trace 3 it under-estimates the IAT autocorrelations. Note in particular, that for traces 1 and 2 the slope of the predicted curve is close to that of the data, which suggests that the correct value of $\hat{H}$ was used.

Figure 7 shows the IAT autocorrelation plots for trace 1 only. The fitted model is represented by lines with symbols, the actual data by a line, and the linear approximation to the data by the straight line without symbols. Also shown on Figure 7 are the predicted IAT autocorrelation functions in cases where the value of $K$ in equations (11) and (12) is either multiplied or divided by a factor of 10 , relative to the fitted value $\mathrm{K}_{0}$ (triangles). The plot in Figure 7 shows that the Pareto model is quite sensitive to the value of $K$. 


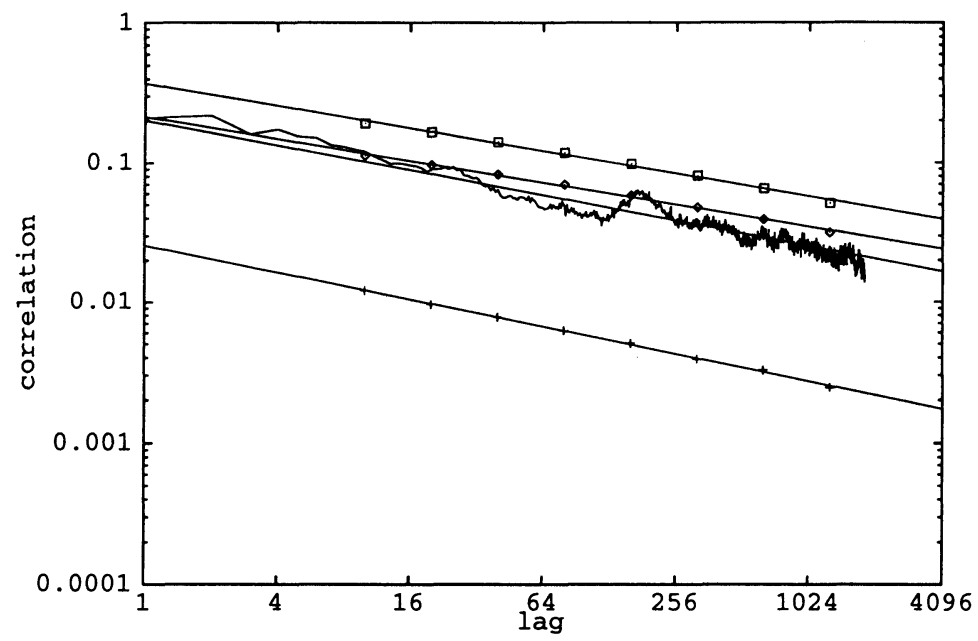

Figure 7 Interarrival time autocorrelation functions for trace 1

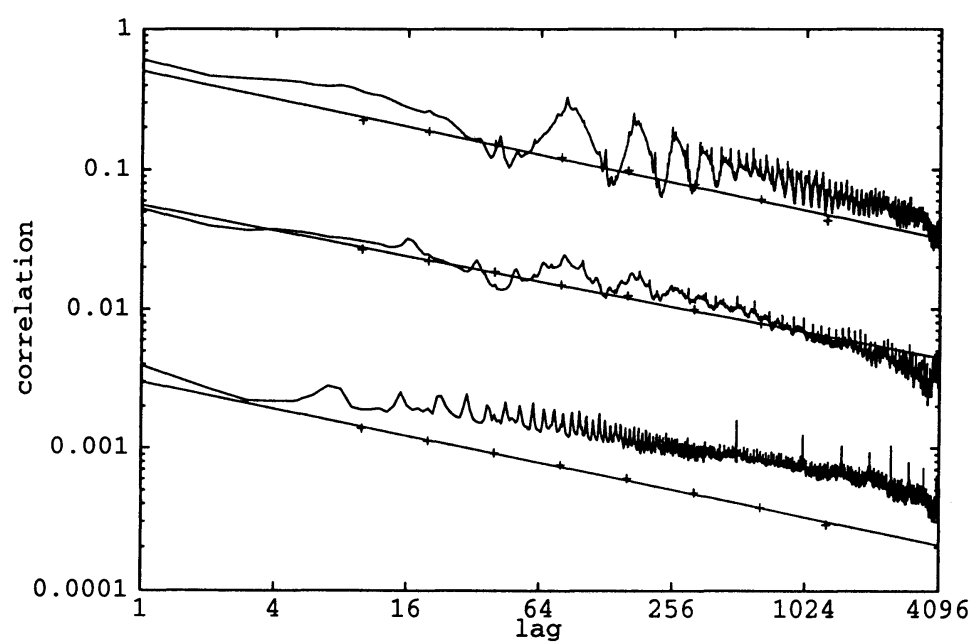

Figure 8 Block packet count autocorrelation functions 
Figure 8 shows the corresponding plots of the BPC autocorrelation functions $\hat{\rho}_{\mathrm{BPC}}(k)$ : symbols represent results derived via simulation of the fitted Pareto model, while the lines without symbols represent the actual data. The model again fits traces 1 and 2 reasonably well, while it under-estimates the BPC autocorrelations for trace 3. The closest match to the actual BPC autocorrelation function appears to be obtained for trace 2, which is also the trace for which the most samples (i.e., arrival events) were collected. The accuracy of the fit in this case may reflect the fact that more data was available in trace 2 .

\section{QUEUEING PERFORMANCE}

Figure 9 shows the mean time averaged queue lengths obtained when each of the three Ethernet traces is used to drive a G/D/1 simulation (dotted lines). These curves were obtained by running the simulation repeatedly, with the service time adjusted to give a specified utilization. Also shown in Figure 9 are the three queue length curves predicted by the fitted Pareto model (solid lines). The Pareto curves under-estimate the mean queue lengths for utilizations less than about 0.7 . At higher utilizations, the Pareto curves rise more steeply than the actual trace driven curves.

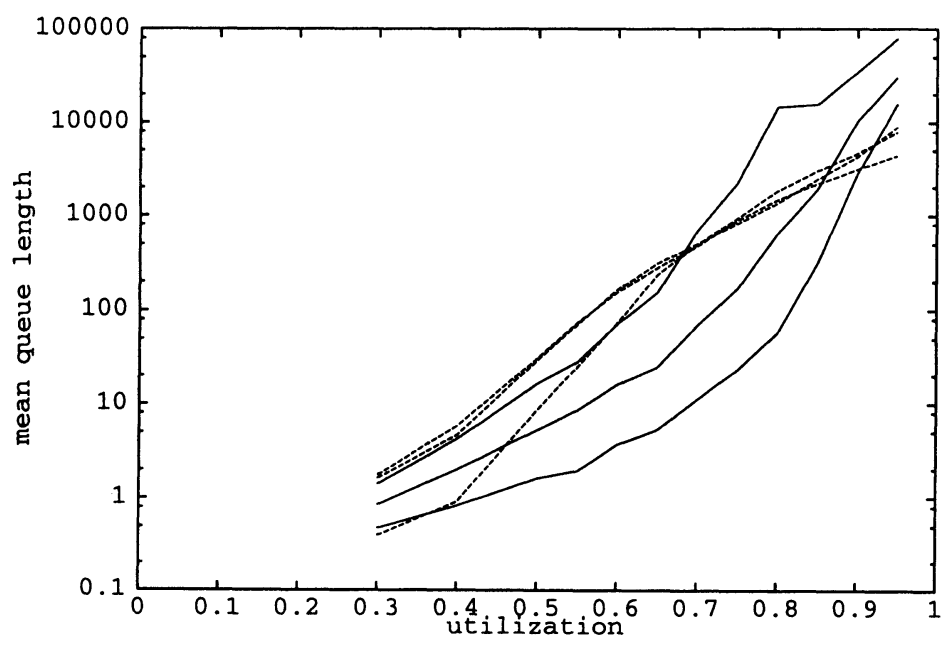

Figure 9 Mean queue length plots

In general, the Pareto model does not closely match the trace driven queue lengths. Note, however, that the $y$-axis on Figure 9 extends over a very large range. Given that the traces each contain only about $10^{6}$ arrivals, one would not expect the curves to be 
accurate in the upper ranges of Figure 9. The traces may contain too few events to accurately predict the probabilities of very large queue lengths.

Another related reason for inaccuracy in Figure 9 may be that the traces are not sufficiently long to average out the large variability inherent in self-similar processes. Figure 10 shows five queue length curves obtained by using five equal parts of trace 1 to drive separate simulations. The queue length results vary significantly within this set, even though they are part of the same trace. The unconnected symbols in Figure 10 represent the overall average queue length for trace 1 .

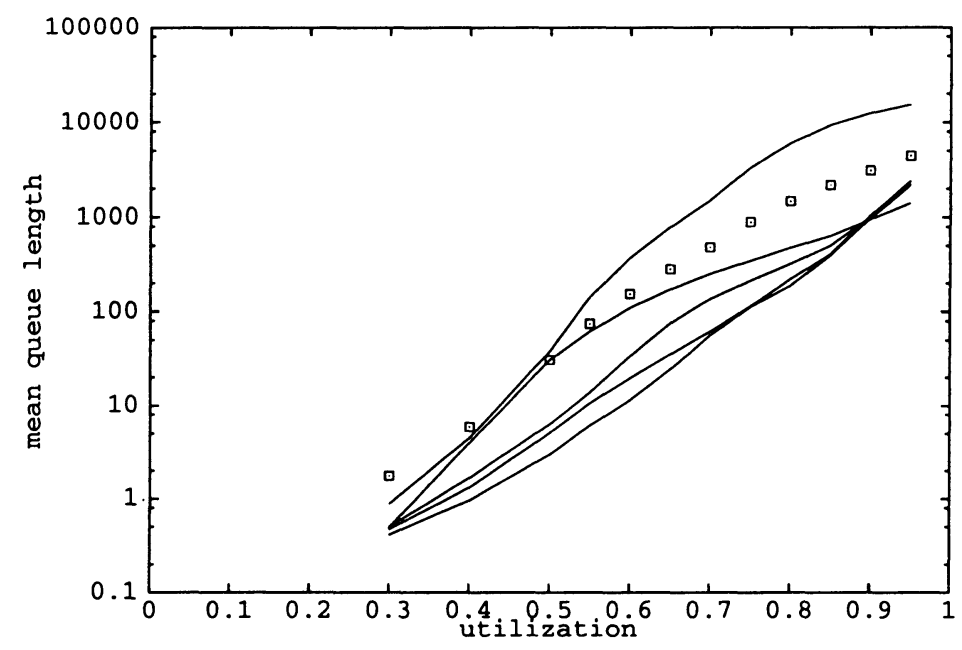

Figure 10 Mean queue length plots for trace 1

For the above reasons, it may be more relevant to check whether the Pareto model accurately predicts the 'knee' in the queue length curve. Figure 11 shows the same plots as in Figure 9, but on a more realistic scale. 


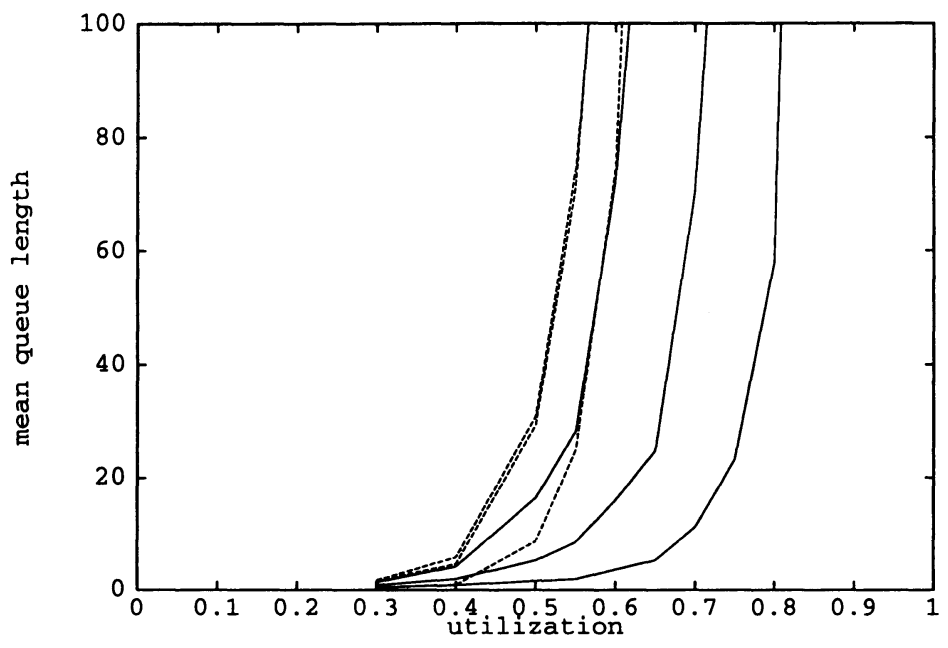

Figure 11 Mean queue length plots

This figure shows that the Pareto model fails to predict the knee in the queue length curves for traces 1 and 3 - it predicts the knee to occur at utilizations around 0.7 rather than 0.5. However, the Pareto model does correctly predict the knee for trace 2 to occur at a utilization of 0.55 .

In conclusion, the length of the Ethernet traces does not permit an accurate decision to be made regarding the Pareto model's effectiveness at predicting queueing behavior. It appears that significantly longer traces are required in order to reach definite conclusions. However, the Pareto model does accurately predict the knee in the queue length curve for trace 2 , and it is possible that the model is successful in this case because it accurately matches the BPC correlation structure in Figure 8. If this observation is correct, the proposed Pareto model has the potential to accurately predict queueing behavior if it is fitted to the data in such a way as to provide a closer match with $\hat{\rho}_{\mathrm{BPC}}(k)$. This issue is the subject of further research.

\section{CONCLUSIONS}

This paper proposes a simple four-parameter model of self-similar data traffic. The basic element of the model is a single Pareto arrival stream, in which packet interarrival times are iid Pareto distributed. The model proposes that two such streams be multiplexed or superimposed, to produce a composite stream in which the marginal IAT distribution is Pareto-like, and which exhibits power law IAT and BPC correlations. Based on analysis of three Ethernet traces collected by Bellcore, the 
proposed model is in good qualitative agreement with observed marginal IAT distributions, and with the corresponding IAT and BPC autocorrelation functions.

In simulated queueing studies, the proposed model accurately reproduced the 'knee' in the mean delay curve for one Ethernet trace. It did not accurately predict the queueing behavior of the other two traces. However, this may have been due to shortcomings of the proposed parameter estimation method, rather than shortcomings of the model itself. Further research into parameter estimation techniques, and / or analysis of longer Ethernet traces, is necessary in order to reach definite conclusions regarding the proposed model's effectiveness at predicting queueing behavior.

In summary, the proposed model exhibits the qualitative features present in selfsimilar Ethernet traffic: Pareto-like IAT marginals, and power law IAT and BPC correlations. The model is conceptually simple, and easy to work with numerically (i.e., to simulate). It provides physical insight into the nature of long range correlations, and the relationship between IAT and BPC correlations. Although not emphasized in the body of the paper, the model can fit non-self-similar as well as selfsimilar arrival processes. In the case of non-self-similar arrivals, the fitted Pareto streams will have larger $\alpha$ values (i.e., Hurst parameter is equal to 0.5 ), and the power law tail of the IAT distribution will decay more rapidly, so it more closely resembles exponential decay. A multiplexed Pareto model therefore has the potential to be a unified model of both self-similar and non-self-similar traffic.

The model proposed here can be generalized in several directions. For example, one could multiplex more than two streams (see [2] for a discussion of this idea). Refinement of the Pareto model is a subject of ongoing research.

\section{REFERENCES}

[1] J.J. Gordon, Pareto Process as a Model of Self-Similar Packet Traffic, Proc. Globecom '95, Singapore 1995, pp. 2232-2236.

[2] J.J. Gordon, Long Range Correlation in Multiplexed Pareto Traffic, Proc. Broadband Communications '96, Montreal, Canada 1996, pp. 28-39.

[3] W.S. Leland, W. Willinger, M.S. Taqqu, D.V. Wilson, Statistical Analysis and Stochastic Modeling of Self-Similar Datatraffic, Proc. 14th International Teletraffic Congres, Juan Les Pins, France 1994, pp. 319-328.

[4] O. Rose, Estimation of the Hurst Parameter of Long Range Dependent Time Series, Research Report No. 137, University of Würzburg, January, 1996.

[5] I. Norros, On the Use of Fractional Brownian Motion in the Theory of Connectionless Networks, IEEE JSAC, Vol. 13 No. 6, pp. 953-962. 
[6] B.K. Ryu, S.B. Lowen, Point Process Approaches to the Modeling and Analysis of Self-Similar Traffic - Part 1: Model Construction, Proc. Infocom '96, San Francisco, U.S.A., pp. 1468-1475.

[7] P.R. Jelenkovic, A.A. Lazar, N. Semret, Multiple Time Scales and Subexponentiality in MPEG Video Streams, Proc. Broadband '96, Montreal, Canada, pp. 64-75.

[8] N. Likhanov, B. Tsybakov, N.D. Georganas, Analysis of an ATM Buffer with Self-Similar ("Fractal") Input Traffic, Proc. Infocom '95, Boston, U.S.A.

[9] L. Kleinrock, Queueing Systems, Volume 1, Wiley 1975.

\section{BIOGRAPHIES}

J.J. Gordon holds B.Sc. and Ph.D. degrees from the Universities of Queensland and Tasmania, Australia. Since completing his Ph.D. in 1987, he has held positions as Research Assistant in the Centre for Teletraffic Research at the University of Adelaide, Australia, Assistant Professor of Teletraffic Science in the School of Information and Computing Sciences at Bond University, Australia, and Member of Technical Staff at Bell Communications Research (Bellcore), New Jersey, U.S.A. Since April 1997, J.J. Gordon has been a consultant for CAPE Consulting, New Jersey, U.S.A., focusing on capacity and performance engineering of telecommunications networks. He can be contacted at www.capecons.com or by sending email to jjg@capecons.com.

Dr. Frank Huebner-Szabo de Bucs received his M.S. degree in Computer Science from the University of Erlangen/Nuernberg in Germany in 1989 and his Ph.D. degree from the University of Wuerzburg in Germany in 1993. Since then, he has been working for Telecom Australia Research Laboratories in Melbourne, Australia and Bell Communications Research in Red Bank, N.J., U.S.A. In January 1997 he joined the "Teletraffic and Performance Analysis Department" at AT\&T Labs in Holmdel, N.J. He participated between 1991 and 1994 as an expert in the European COST 242 project "Methods for Performance Evaluation and Design of Multi-Service Broad Band Networks". He is a permanent member of the program committee of the IEEE Local Computer Networks Conference and program co-chairman of that conference in 1998. He is working in the area of performance evaluation of queueing systems, with special emphasis on discrete-time queueing systems and congestion control issues. His main topics of interest are modeling of data traffic, performance analysis of the Internet, ATM systems, manufacturing systems, and queueing systems in general. 